\title{
Architectural Singularities of a Class of Pentapods
}

\author{
Júlia Borràs, Federico Thomas, and Carme Torras \\ Institut de Robòtica i Informàtica Industrial (CSIC-UPC) \\ Llorens Artigues 4-6, 08028 Barcelona, Spain \\ \{jborras,fthomas,ctorras\}@iri.upc.edu
}

\begin{abstract}
A pentapod is usually defined as a 5-degrees-of-freedom fully-parallel manipulator with an axial spindle as moving platform. This kind of manipulators has revealed as an interesting alternative to serial robots handling axisymmetric tools. Their particular geometry permits that, in one tool axis, high inclination angles could be attained, thus overcoming the orientation limits of the classical Stewart-Gough platform. This paper deals with pentapods with coplanar base attachments.

In previous works changes in the location of the leg attachments that do not modify the singularity locus of the pentapod were studied. Such leg rearrangements reveal here as a powerful tool to shed light on the geometric structure of the singularity locus and, in particular, on architectural singularities.

Indeed, a complete analysis of such singularities is carried out, providing both algebraic conditions, which complete previous results found in literature, and a geometrical interpretation that permits defining a measure of distance to architectural singularities. Such measure can be used as a index in the design process to obtain manipulators as far as possible from architectural singularities, leading to a better global behavior.
\end{abstract}

Keywords: Parallel manipulators, robot kinematics, architectural singularities, singularities, manipulator design.

\section{Introduction}

The Stewart-Gough platform is defined as a 6-DoF parallel mechanism with six identical UPS legs [1], [2]. Although it is certainly the most celebrated parallel mechanism, platforms with a lower number of UPS legs are also of interest both from the theoretical and practical points of view. Kong and Gosselin refer to them as components as they can always be considered as rigid subassemblies in a standard Stewart-Gough platform [3].

A parallel robot consisting of a base and a moving platform connected by five SPU legs is clearly uncontrollable. For example, if the universal joints are aligned as in Fig. 1 , the moving platform can freely rotate around the axis defined by these five aligned universal joints. Nevertheless, observe that in this particular case the uncontrolled motion is irrelevant if the rotation axis is made coincident with the symmetry axis of the tool. Indeed, there are important industrial tasks requiring a tool to be perpendicular to a $3 \mathrm{D}$ free-from surface along a given trajectory without caring about its axial orientation. 


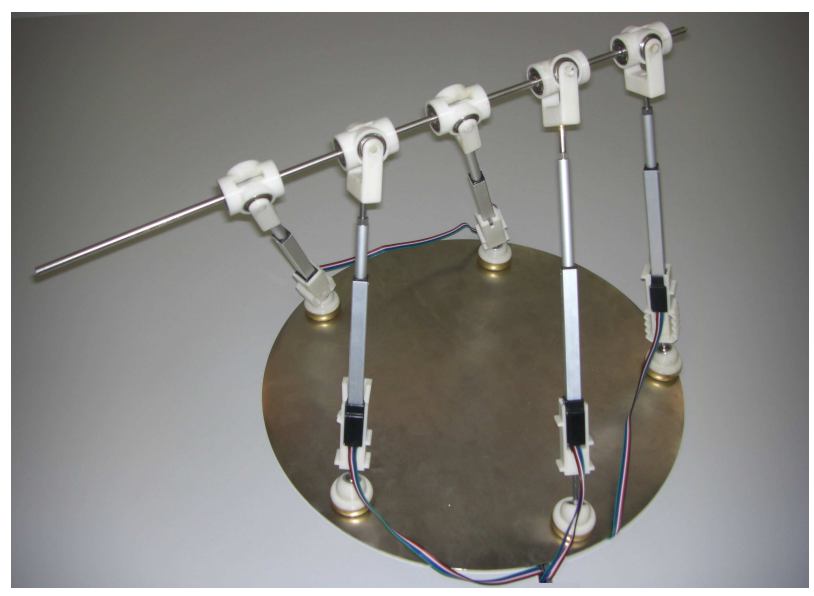

Figure 1: Prototype of a pentapod with coplanar base attachments.

They include, for example, 5-axis milling, laser-engraving, spray-based painting, surface polishing and water-jet cutting. The study of the kinematics properties of 5-SPU parallel robots, or pentapods, is thus highly relevant for many applications $[4,5,6]$.

Zhang and Song were the first to solve the forward kinematics of a general GoughStewart platform containing a five-legged component with collinear attachments in the platform and coplanar in the base [7]. They showed how the line defined by the five attachments in the platform can attain, in the general case, up to eight configurations with respect to the base plane for a given set of leg lengths.

A singularity analysis of this class of pentapods with planar base unraveled that there are only three general topologies for their singularity locus, corresponding to the families of quartically-, cubically- and quadratically-solvable 5-SPU parallel robots [8]. The members of the last family have only four assembly modes, which are obtained by solving two quadratic equations [9]. Moreover, it was shown that the parallel singularities of all three families obey a neat structure, whose unified geometric interpretation simplifies control strategies to avoid them at runtime. In the current paper, we extend the characterization of the architectural singularities for the members of the quadratically-solvable family presented in [9] to the other two families.

The widely accepted use of the term architectural singularity refers to a robot design that is singular in all the points of the workspace, although its first definition, appeared in 1992 in Ma and Angeles work [10], required it to be singular only in a region of the workspace. Later this was considered a regular singularity, since it only occurs for particular poses of the platform [11, 12, 13]. Architecturally singular manipulators are useless, since they cannot be controlled. Thus, their characterization is important to avoid them in the early design process.

Before parallel robots even existed, this problem was first studied in 1851 by M. Chasles [14], as how six bars can support a rigid body. He stated that they cannot support it if the axes of the bars belong to a linear line complex [15]. Lately this problem was also analyzed by mathematicians like E. Borel [16] and R.Bricard [17], who classified all the self-motions that such structures exhibit when the six bars cannot support the 


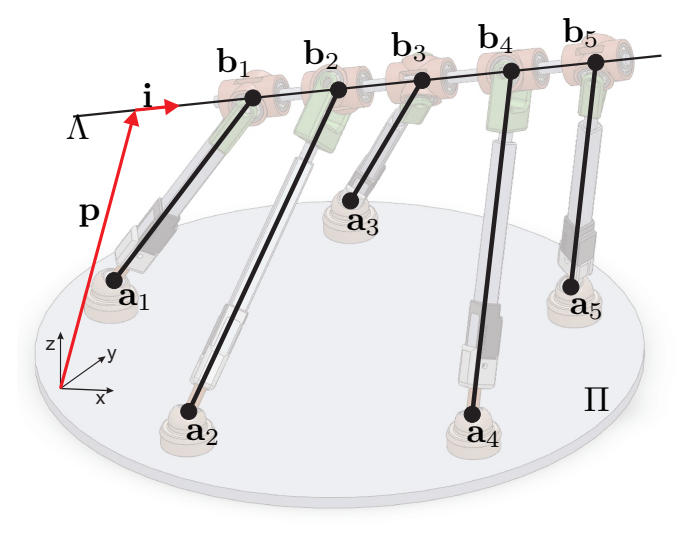

Figure 2: Schematic representation of a pentapod, a 5-S $\underline{P U}$ parallel robot.

rigid body. These publications can be directly related to architectural singularities in parallel platforms. Indeed, following Borel and Bricard's work, Husty and Karger studied the conditions for a 5-UPS platform to be architecturally singular and found two algebraic conditions that must be simultaneously satisfied [18, 19, 20].

Borràs and Thomas recently showed that, if the attachments on the base plane are moved along a particular set of lines that form a pencil, the singularities of the platform remain unaltered [21]. Although this does not solve the singularity characterization problem, it provides a lot of insight into its nature. Indeed, in this work it will be shown how such singularity-invariant leg rearrangements reveal a hidden geometric structure that allows to geometrically characterize architectural singularities, generalizing the previous result found by Husty and Karger, and giving a measure of distance to such singularities. Such measure of distance can be useful to design manipulators as far as possible from such singularities, thus providing a better global behavior.

The paper is organized as follows. Section 2 uses a previous characterization of the pentapod singularities to describe the singularity-invariant leg rearrangements that define the underlying geometric structure of the 5-SPU. Architectural singularities and a measure of distance to them are introduced in Section 3, and then Section 4 presents several examples. Finally, Section 5 summarizes the main contributions.

\section{Geometric structure of the singularities}

Let us consider the pentapod appearing in Fig. 2, whose base and platform attachments lay on plane $\Pi$ and line $\Lambda$, respectively. Let $\Pi$ coincide with the $x y$-plane of the base reference frame. Thus, the leg attachments in the base have coordinates $\mathbf{a}_{i}=\left(x_{i}, y_{i}, 0\right)^{T}$, for $i=1, \ldots, 5$. The pose of $\Lambda$ with respect to $\Pi$ can be described by the position vector $\mathbf{p}=\left(p_{x}, p_{y}, p_{z}\right)^{T}$ and the unit vector $\mathbf{i}=(u, v, w)^{T}$ in the direction of $\Lambda$. Thus, the coordinates of the leg attachments in platform $\Lambda$, expressed in the base reference frame, can be written as $\mathbf{b}_{i}=\mathbf{p}+z_{i} \mathbf{i}$. For the sake of simplicity, we use the same symbol to denote a point and its position vector.

In previous works, the authors have been successful to characterize the singularities of such pentapods in terms of the determinant of a new matrix [21]. After computing 
the non-normalized Jacobian matrix, whose columns are the Plücker coordinates of the leg lines, and factorizing its determinant, the singularities of a 5-SPU are defined by

$$
\operatorname{det}(\mathbf{T})=0
$$

where $\mathbf{T}$ is the matrix

$\mathbf{T}=\left(\begin{array}{cccccc}w p_{z} & w\left(p_{z} u-p_{x} w\right) & w\left(p_{z} v-p_{y} w\right) & p_{z}\left(p_{x} w-p_{z} u\right) & p_{z}\left(p_{y} w-p_{z} v\right) & -w^{2} \\ z_{1} & x_{1} & y_{1} & x_{1} z_{1} & y_{1} z_{1} & 1 \\ z_{2} & x_{2} & y_{2} & x_{2} z_{2} & y_{2} z_{2} & 1 \\ z_{3} & x_{3} & y_{3} & x_{3} z_{3} & y_{3} z_{3} & 1 \\ z_{4} & x_{4} & y_{4} & x_{4} z_{4} & y_{4} z_{4} & 1 \\ z_{5} & x_{5} & y_{5} & x_{5} z_{5} & y_{5} z_{5} & 1\end{array}\right)$.

In what follows, let $\hat{\mathbf{T}}$ denote the $5 \times 6$ matrix resulting from removing the first row of T. It is important to realize that this matrix depends only on the coordinates of the leg attachments, whereas the first row of $\mathbf{T}$ depends on the pose of the manipulator. This leads to a clear-cut distinction of two types of singularities, architectural and parallel, the former depending exclusively on $\hat{\mathbf{T}}$, whereas the latter depend also on the pose.

For non-architecturally-singular manipulators, i.e., those for which $\hat{\mathbf{T}}$ is not rank defective, the condition $\operatorname{det}(\mathbf{T})=0$ characterizes the set of robot poses lacking stiffness. Expanding $\operatorname{det}(\mathbf{T})$ by its first row, the following polynomial equation is obtained:

$C_{1} w p_{z}+C_{2} w\left(p_{z} u-p_{x} w\right)+C_{3} w\left(p_{z} v-p_{y} w\right)+C_{4} p_{z}\left(p_{x} w-p_{z} u\right)+C_{5} p_{z}\left(p_{y} w-p_{z} v\right)-C_{6} w^{2}=0$,

where $C_{i}$ for $i=1, \ldots, 5$ are the cofactors of the elements of the first row of $\mathbf{T}$. This defines the singularity hypersurface in the configuration space of the pentapod.

The attachments of the $i$-th leg can be determined by a single point in $\mathbb{R}^{3}$ with coordinates $\left(x_{i}, y_{i}, z_{i}\right)$. This $3 D$ space of leg attachments plays an important role in the definition of leg rearrangements. Let us consider the following surface in this space of leg attachments:

$$
\left\{(x, y, z) \in \mathbb{R}^{3}|| \begin{array}{cccccc}
z & x & y & x z & y z & 1 \\
z_{1} & x_{1} & y_{1} & x_{1} z_{1} & y_{1} z_{1} & 1 \\
z_{2} & x_{2} & y_{2} & x_{2} z_{2} & y_{2} z_{2} & 1 \\
z_{3} & x_{3} & y_{3} & x_{3} z_{3} & y_{3} z_{3} & 1 \\
z_{4} & x_{4} & y_{4} & x_{4} z_{4} & y_{4} z_{4} & 1 \\
z_{5} & x_{5} & y_{5} & x_{5} z_{5} & y_{5} z_{5} & 1
\end{array} \mid=0\right\}
$$

The last five rows of the determinant above coincide with $\hat{\mathbf{T}}$, thus the coefficients of the polynomial resulting from expanding the determinant by its first row coincide with those in $(3)$ :

$$
C_{1} z+C_{2} x+C_{3} y+C_{4} x z+C_{5} y z+C_{6}=0,
$$

which in turn can be rewritten in vector form as:

$$
\left.\left[\begin{array}{lll}
\left(C_{2}\right. & C_{3} & C_{6}
\end{array}\right)+z\left(\begin{array}{lll}
C_{4} & C_{5} & C_{1}
\end{array}\right)\right]\left(\begin{array}{l}
x \\
y \\
1
\end{array}\right)=0 .
$$




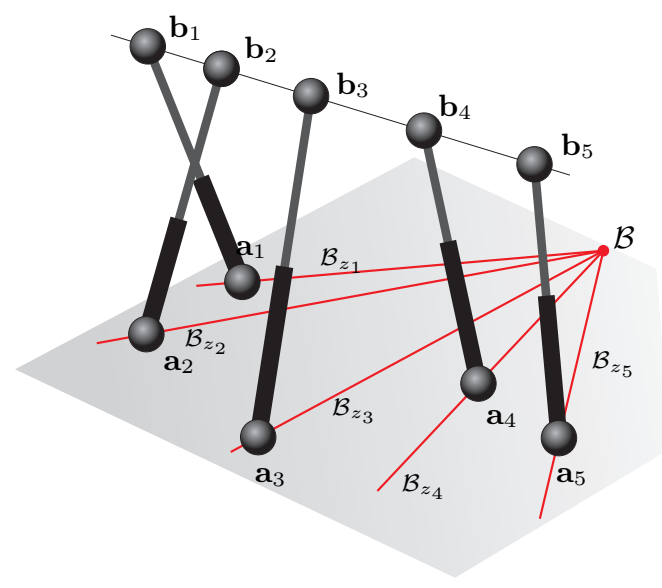

Figure 3: There is a one-to-one correspondence between the attachments in the platform line, $\Lambda$, and the lines of the pencil centered at $\mathcal{B}$.

Hence, if any of the pentapod legs is substituted by a new leg with attachments $(x, y, z)$ satisfying $(6)$, then the coefficients of the polynomial in (3) will be the same up to a constant multiple. In other words, its roots will be the same and, therefore, the singularity locus of the pentapod will not change. In conclusion, equation (6) defines singularity-invariant leg rearrangements.

The same implicit equation (5) was deduced [22] by imposing that the squared leg lengths before and after a leg rearrangement - for an arbitrary fixed pose of the moving platform - are affinely related. This kind of reconfigurations keeps not only the singularity locus unchanged, but also the resulting kinematic equations are essentially the same.

Equation (6) can be interpreted geometrically as a correspondence between points in $\Lambda$ and lines of a pencil embedded in $\Pi$ (refer to Fig. 3). Indeed, a point in $\Lambda$ with coordinate $z$ determines a line $\mathcal{B}_{z}$ in $\Pi$ and, by varying $z$, a pencil of lines with focus located at:

$$
\mathcal{B}=\left(\frac{C_{3} C_{1}-C_{6} C_{5}}{C_{2} C_{5}-C_{4} C_{3}},-\frac{C_{2} C_{1}-C_{4} C_{6}}{C_{2} C_{5}-C_{4} C_{3}}, 0\right)
$$

is obtained. On the way round, each line of the pencil, i.e., a line through $\mathcal{B}$ and $(x, y, 0)$, determines a point in $\Lambda$ with coordinate

$$
z=-\frac{C_{2} x+C_{3} y+C_{6}}{C_{4} x+C_{5} y+C_{1}} .
$$

Note that the same $z$ value is obtained for all the points $(x, y, 0)$ on the same line through $\mathcal{B}$.

In what follows, a line of the pencil will be called $\mathcal{B}$-line, and the one-to-one correspondence between points in $\Lambda$ and $\mathcal{B}$-lines in $\Pi$, defined by equation (6), will be called $\mathcal{B}$ correspondence. This geometric structure is revealed when studying singularity-invariant 
leg rearrangements, but it provides a lot of information for the geometrical characterization of the pentapod singularities. Indeed, in the following sections it will be shown how point $\mathcal{B}$ is strongly related to the characterization of architectural singularities.

\section{Architectural singularities}

\subsection{Algebraic characterization}

For the sake of simplicity, we will assume that we are dealing with generic cases, that is, the attachments are in general position. As a consequence, none of the base attachments is coincident with $\mathcal{B}$. The present methodology can also deal with nongeneric cases, but they must be studied separately (see [23] for details).

When $\hat{\mathbf{T}}$ is rank defective, $\operatorname{det}(\mathbf{T})$ is identically zero irrespective of the pose of $\Lambda$ with respect to $\Pi$ and, hence, the pentapod is said to be architecturally singular [10].

If, to check rank defectiveness, Gaussian elimination is applied on $\hat{\mathbf{T}}$, the last row of the resulting matrix is

$$
\frac{1}{D_{5,6}}\left(\begin{array}{llllll}
0 & 0 & 0 & 0 & -C_{6} & C_{5}
\end{array}\right)
$$

where $C_{i}$ are the cofactors of the first row of $\mathbf{T}$ as in equation (3), and $D_{i, j}$ is the determinant of the matrix formed by the first four rows of $\hat{\mathbf{T}}$ with the $i$ th and $j$ th columns removed.

By permuting the columns of $\hat{\mathbf{T}}$, we can conclude that a necessary and sufficient condition for a pentapod to be architecturally singular is that

$$
\exists i, j \in\{1, \ldots, 6\}, i \neq j \text {, such that } C_{i}=C_{j}=0 \text { and } D_{i, j} \neq 0 .
$$

Note that the conditions $C_{4}=C_{5}=0$ are in one-to-one correspondence with the two algebraic conditions given by Husty and Karger [18, Theorem 1.6] (after setting $x_{1}=y_{1}=z_{1}=y_{2}=0$, which can always be done without loss of generality by properly placing the reference frames). When the attachments are in general position, $D_{4,5} \neq 0$, and thus Husty and Karger's conditions are equivalent to (10). However, for platforms whose attachments are not in a generic position (such as, for instance, the quadratically solvable manipulators presented in [9]), it can occur that $C_{4}=C_{5}=D_{4,5}=0$ and the manipulator is not architecturally singular (see Section 4.3 for an example).

\subsection{Geometric interpretation}

Is important not only characterizing architectural singularities to avoid them, but also to determine how near the pentapod is to an architecturally singular one in order to improve its design to obtain a better global behavior. With this purpose, a geometric interpretation of condition (10) is next derived.

Suppose that one of the legs, say $l_{5}$, is reconfigured to the new attachments $\mathbf{a}=$ $(x, y, 0)$ and $\mathbf{b}=\mathbf{p}+z \mathbf{i}$. Assuming that $D_{12} \neq 0$, the manipulator becomes architecturally 
singular, according to (10), when $C_{1}$ and $C_{2}$ are zero. This defines the following system of equations in the space of leg attachments:

$$
\left\{\widetilde{C_{1}}=\left|\begin{array}{ccccc}
x & y & x z & y z & 1 \\
x_{1} & y_{1} & x_{1} z_{1} & y_{1} z_{1} & 1 \\
x_{2} & y_{2} & x_{2} z_{2} & y_{2} z_{2} & 1 \\
x_{3} & y_{3} & x_{3} z_{3} & y_{3} z_{3} & 1 \\
x_{4} & y_{4} & x_{4} z_{4} & y_{4} z_{4} & 1
\end{array}\right|=0, \quad \widetilde{C_{2}}=\left|\begin{array}{ccccc}
z & y & x z & y z & 1 \\
z_{1} & y_{1} & x_{1} z_{1} & y_{1} z_{1} & 1 \\
z_{2} & y_{2} & x_{2} z_{2} & y_{2} z_{2} & 1 \\
z_{3} & y_{3} & x_{3} z_{3} & y_{3} z_{3} & 1 \\
z_{4} & y_{4} & x_{4} z_{4} & y_{4} z_{4} & 1
\end{array}\right|=0\right\}
$$

where $\widetilde{C_{i}}$ equals cofactor $C_{i}$ evaluated at $\left\{x=x_{5}, y=y_{5}, z=z_{5}\right\}$. Then, the roots of this system define the locus of the 5th leg attachments that make the manipulator architecturally singular. By expanding the determinants in (11) by their first rows, the above system results in:

$$
\left.\begin{array}{l}
D_{1,2} x+D_{1,3} y+D_{1,4} z x+D_{1,5} z y+D_{1,6}=0 \\
D_{1,2} z+D_{2,3} y+D_{2,4} z x+D_{2,5} z y+D_{2,6}=0
\end{array}\right\}
$$

which can be rewritten as

$$
\left(\begin{array}{cc}
D_{1,4} x+D_{1,5} y & D_{1,2} x+D_{1,3} y+D_{1,6} \\
D_{2,4} x+D_{2,5} y+D_{1,2} & D_{2,3} y+D_{2,6}
\end{array}\right)\left(\begin{array}{l}
z \\
1
\end{array}\right)=\left(\begin{array}{l}
0 \\
0
\end{array}\right),
$$

which clearly has a solution for $z$ if, and only if,

$$
\left|\begin{array}{cc}
D_{1,4} x+D_{1,5} y & D_{1,2} x+D_{1,3} y+D_{1,6} \\
D_{2,4} x+D_{2,5} y+D_{1,2} & D_{2,3} y+D_{2,6}
\end{array}\right|=0 .
$$

In conclusion, there exists a value for $z$ that satisfies (11) only for the points on the conic

$$
\mathcal{C}_{5}=\left\{(x, y) \mid n_{1} x^{2}+n_{2} x y+n_{3} y^{2}+n_{4} x+n_{5} y+n_{6}=0\right\},
$$

where

$$
\begin{aligned}
& n_{1}=D_{1,2} D_{2,4} \\
& n_{2}=D_{2,3} D_{1,4}-D_{1,2} D_{2,5}-D_{1,3} D_{2,4} \\
& n_{3}=D_{1,3} D_{2,5}-D_{2,3} D_{1,5} \\
& n_{4}=D_{1,2}^{2}+D_{1,6} D_{2,4}-D_{2,6} D_{1,4} \\
& n_{5}=D_{2,6} D_{1,5}-D_{1,6} D_{2,5}-D_{1,3} D_{1,2} \\
& n_{6}=D_{1,6} D_{1,2} .
\end{aligned}
$$

The other way round, for a given value of $z$, system (13) gives a point on this conic. In other words, system (13) defines a one-to-one correspondence between the points in $\Lambda$ and the points of conic $\mathcal{C}_{5}$ embedded in $\Pi$.

Actually, five different one-to-one correspondences of the same type exist: the correspondences between points in $\mathcal{C}_{i}$ and points in $\Lambda$, where $\mathcal{C}_{i}$ is the conic that contains all base attachments but $\mathbf{a}_{i}$. Each of these correspondences will be called $\mathcal{C}_{i}$-correspondence and the coordinates of $\mathbf{a}_{j}$ and $\mathbf{b}_{j}$, for $j \in\{1, \ldots, 5\} \backslash\{i\}$, satisfy it by construction. 


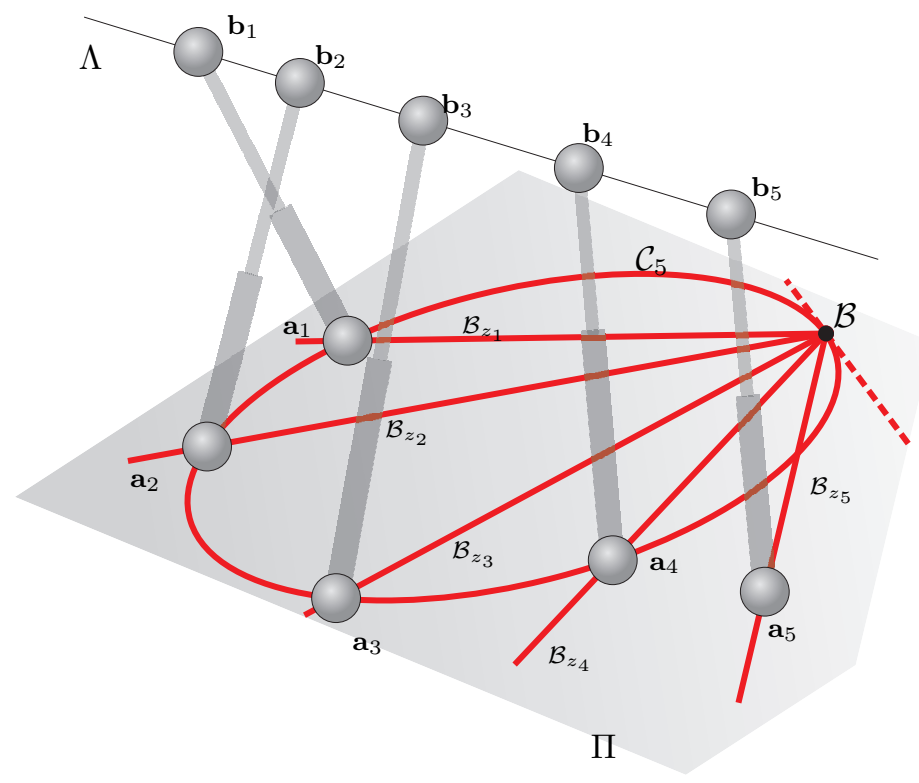

Figure 4: The composition of the one-to-one $\mathcal{B}$-correspondence between points on $\Lambda$ and $\mathcal{B}$-lines on $\Pi$, and the one-to-one $\mathcal{C}_{5}$-correspondence between points on $\Lambda$ and points of conic $\mathcal{C}_{5}$. The point on $\Lambda$ corresponding to the point on the conic coincident with $\mathcal{B}$ necessarily corresponds to the $\mathcal{B}$-line tangent to $\mathcal{C}_{5}$ on $\mathcal{B}$.

When the $i$ th leg attachments coordinates also satisfy it, the manipulator becomes architecturally singular. In this particular case, all conics collapse into a single conic, which can be simply expressed as

$$
\mathcal{C}=\left\{(x, y)|| \begin{array}{cccccc}
x^{2} & x y & y^{2} & x & y & 1 \\
x_{1}^{2} & x_{1} y_{1} & y_{1}^{2} & x_{1} & y_{1} & 1 \\
x_{2}^{2} & x_{2} y_{2} & y_{2}^{2} & x_{2} & y_{2} & 1 \\
x_{3}^{2} & x_{3} y_{3} & y_{3}^{2} & x_{3} & y_{3} & 1 \\
x_{4}^{2} & x_{4} y_{4} & y_{4}^{2} & x_{4} & y_{4} & 1 \\
x_{5}^{2} & x_{4} y_{5} & y_{5}^{2} & x_{5} & y_{5} & 1
\end{array} \mid=0\right\}
$$

because five points on a plane define a conic.

\subsection{Proximity to architectural singularities}

Conics $\mathcal{C}_{i}$ can be used to evaluate the proximity of a pentapod to an architectural singularity, but the resulting algebraic expressions are quite involved. To obtain a deeper geometric insight into the problem, it is necessary to study the relation between the $C_{i^{-}}$ correspondences given by equation (13) and the $\mathcal{B}$-correspondence defined by (6).

Let us define the following composition:

$$
\begin{array}{ccccc}
\mathcal{C}_{i} & \mathcal{C}_{i} \text {-correspondence } & \Lambda & \stackrel{\mathcal{B} \text {-correspondence }}{\leftrightarrows} & \text { Pencil in } \Pi \\
(x, y) & \leftrightarrows & z & \leftrightarrows & \mathcal{B}_{z} \\
& & & &
\end{array}
$$


First of all, note that any $(x, y, z)$ satisfying the $\mathcal{C}_{i}$-correspondence satisfies also the $\mathcal{B}$-correspondence, i.e., any point $(x, y, 0) \in \mathcal{C}_{i}$ lies in its corresponding B-line $B_{z}$. This is because, particularizing to $\mathcal{C}_{5}$, (11) implies that two cofactors of the elements of the last row of the matrix in (4) are zero, which makes its determinant zero irrespective of the values given to $\left(x_{5}, y_{5}, z_{5}\right)$. Therefore, the $z$ coordinate corresponding to $(x, y, 0)$ on the conic $\mathcal{C}_{i}$ is the same as the one corresponding to the $\mathcal{B}_{z}$-line through $(x, y, 0)$ (in Fig. 4 there is a graphical representation of the $\mathcal{C}_{5}$-correspondence and the $\mathcal{B}$-correspondence).

As the composition of two one-to-one correspondences is one-to-one, there is a oneto-one correspondence between points in $\mathcal{C}_{i}$ and lines of the pencil embedded in $\Pi$. This fact has two consequences:

1. Point $\mathcal{B}$ lies on the conic, otherwise there would be two points of the conic corresponding to the same $\mathcal{B}$-line.

2. Point $\mathcal{B}$ has a unique corresponding point in $\Lambda$ through the $\mathcal{C}_{i}$-correspondence, which is necessarily the point corresponding to the $\mathcal{B}$-line tangent to conic $\mathcal{C}_{i}$ on $\mathcal{B}$ through the $\mathcal{B}$-correspondence.

As a consequence, a generic pentapod is architecturally singular when the base attachment $\mathbf{a}_{i}$ lies on conic $\mathcal{C}_{i}$ defined by the attachments of the other four legs and $\mathcal{B}$. This observation is of practical interest to derive a distance to an architectural singularity: the distance from point $\mathcal{B}$ to the conic defined in (15). This distance can be evaluated by substituting $(x, y)$ in the conic expression:

$$
\mathcal{C}\left(b_{x}, b_{y}\right)=\left|\begin{array}{llllll}
b_{x}^{2} & b_{x} b_{y} & b_{y}^{2} & b_{x} & b_{y} & 1 \\
x_{1}^{2} & x_{1} y_{1} & y_{1}^{2} & x_{1} & y_{1} & 1 \\
x_{2}^{2} & x_{2} y_{2} & y_{2}^{2} & x_{2} & y_{2} & 1 \\
x_{3}^{2} & x_{3} y_{3} & y_{3}^{2} & x_{3} & y_{3} & 1 \\
x_{4}^{2} & x_{4} y_{4} & y_{4}^{2} & x_{4} & y_{4} & 1 \\
x_{5}^{2} & x_{4} y_{5} & y_{5}^{2} & x_{5} & y_{5} & 1
\end{array}\right|,
$$

where $\mathcal{B}=\left(b_{x}, b_{y}, 0\right)$.

\section{Examples}

In this section several examples will illustrate the presented theoretical results. The first example shows how an architecturally singular manipulator can be obtained, with the computation of its associated self-motion, and the following two ones show manipulator designs as far as possible from architectural singularities. ${ }^{1}$

\subsection{An architecturally singular pentapod}

Consider a pentapod with the attachments coordinates appearing in the table of the Fig. 5, with a sketch of such manipulator.

\footnotetext{
${ }^{1}$ This paper has supplementary downloadable multimedia material. This material includes a video and several Maple Worksheets. The Maple Worksheets explain in detail the derivation of the architecturally singular manipulator in Section 4.1 and the examples in Sections 4.2 and 4.3. No special software is required, except for an installed copy of Maple, version 10.0 or higher. Contact Júlia Borràs for further questions about this material.
} 


\begin{tabular}{|c|c|c|c|}
\hline $\mathrm{i}$ & $x_{i}$ & $y_{i}$ & $z_{i}$ \\
\hline \hline 1 & 0 & 2 & 0 \\
\hline 2 & $-3 / 2$ & $9 / 4$ & 1 \\
\hline 3 & -3 & 1 & 2 \\
\hline 4 & -1 & 0 & 3 \\
\hline 5 & -1 & -1 & 4 \\
\hline
\end{tabular}

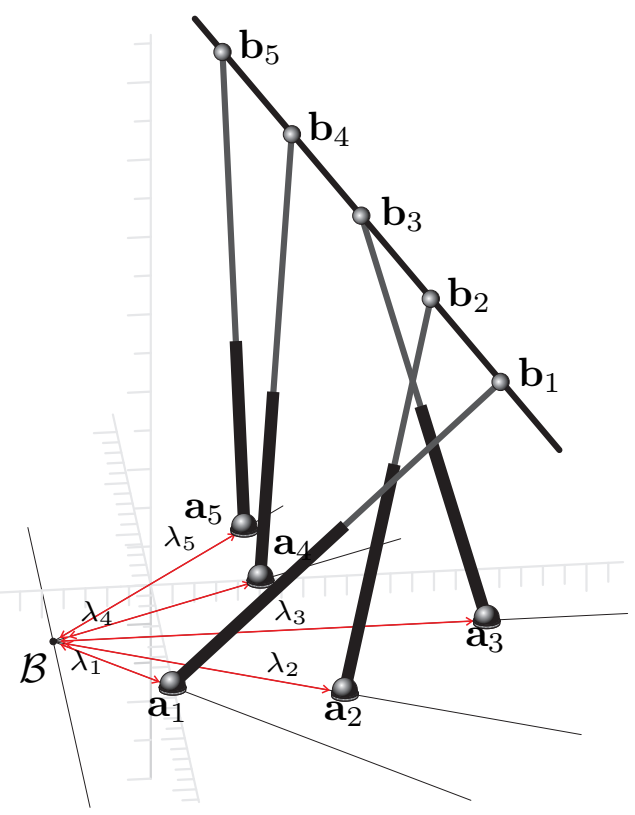

Figure 5: Coordinates of the attachments $\left[\mathbf{a}_{i}=\left(x_{i}, y_{i}, 0\right)\right.$ and $\left.\mathbf{b}_{i}=\mathbf{p}+z_{i} \mathbf{i}\right]$ and scheme of the pentapod analyzed in Section 4.1.

Let $\lambda_{i}$ be the distance between $\mathbf{a}_{i}$ and $\mathcal{B}$. Then, for all generic cases, $\lambda_{i} \neq 0$ for $i=1, \ldots, 5$. The coordinates in Fig. 5 correspond to the configuration $\left\{\lambda_{1}=\sqrt{2}, \lambda_{2}=\right.$ $\left.5 \sqrt{5} / 4, \lambda_{3}=4, \lambda_{4}=\sqrt{5}, \lambda_{5}=2 \sqrt{2}\right\}$.

For a generic configuration given by $\left\{\lambda_{1}, \ldots, \lambda_{5}\right\}$, the value of the cofactors of matrix $\mathbf{T}$ are

$$
\begin{array}{lll}
C_{1}=K, & C_{2}=2 K, & C_{3}=2 K, \\
C_{4}=-K, & C_{5}=0, & C_{6}=-4 K
\end{array}
$$

and thus the singularities are given by

$$
\begin{aligned}
& \operatorname{det}(\mathbf{T})=K\left(w p_{z}-2 w\left(p_{x} w-u p_{z}\right)\right. \\
&\left.-2 w\left(p_{y} w-v p_{z}\right)-p_{z}\left(p_{x} w-u p_{z}\right)+4 w^{2}\right),
\end{aligned}
$$

where

$$
\begin{aligned}
K= & \frac{\sqrt{10}}{50}\left(-15 \sqrt{2} \lambda_{1} \lambda_{2} \lambda_{5}+12 \sqrt{5} \lambda_{1} \lambda_{4} \lambda_{2}-15 \sqrt{2} \lambda_{5} \lambda_{1} \lambda_{4}\right. \\
& -45 \lambda_{1} \lambda_{4} \lambda_{3}+8 \sqrt{10} \lambda_{4} \lambda_{2} \lambda_{3}-45 \lambda_{5} \lambda_{2} \lambda_{3}-5 \lambda_{1} \lambda_{2} \lambda_{3} \\
& \left.+12 \sqrt{5} \lambda_{5} \lambda_{4} \lambda_{2}+20 \sqrt{10} \lambda_{5} \lambda_{1} \lambda_{3}-5 \lambda_{5} \lambda_{4} \lambda_{3}\right),
\end{aligned}
$$

which depends only on $\lambda_{1}, \ldots, \lambda_{5}$.

Hence, this example makes clear that modifying the values of $\lambda_{i}$ (i.e., moving the base attachments along their $\mathcal{B}$-lines) does not modify the singularities of the manipulator, provided $K \neq 0$. 


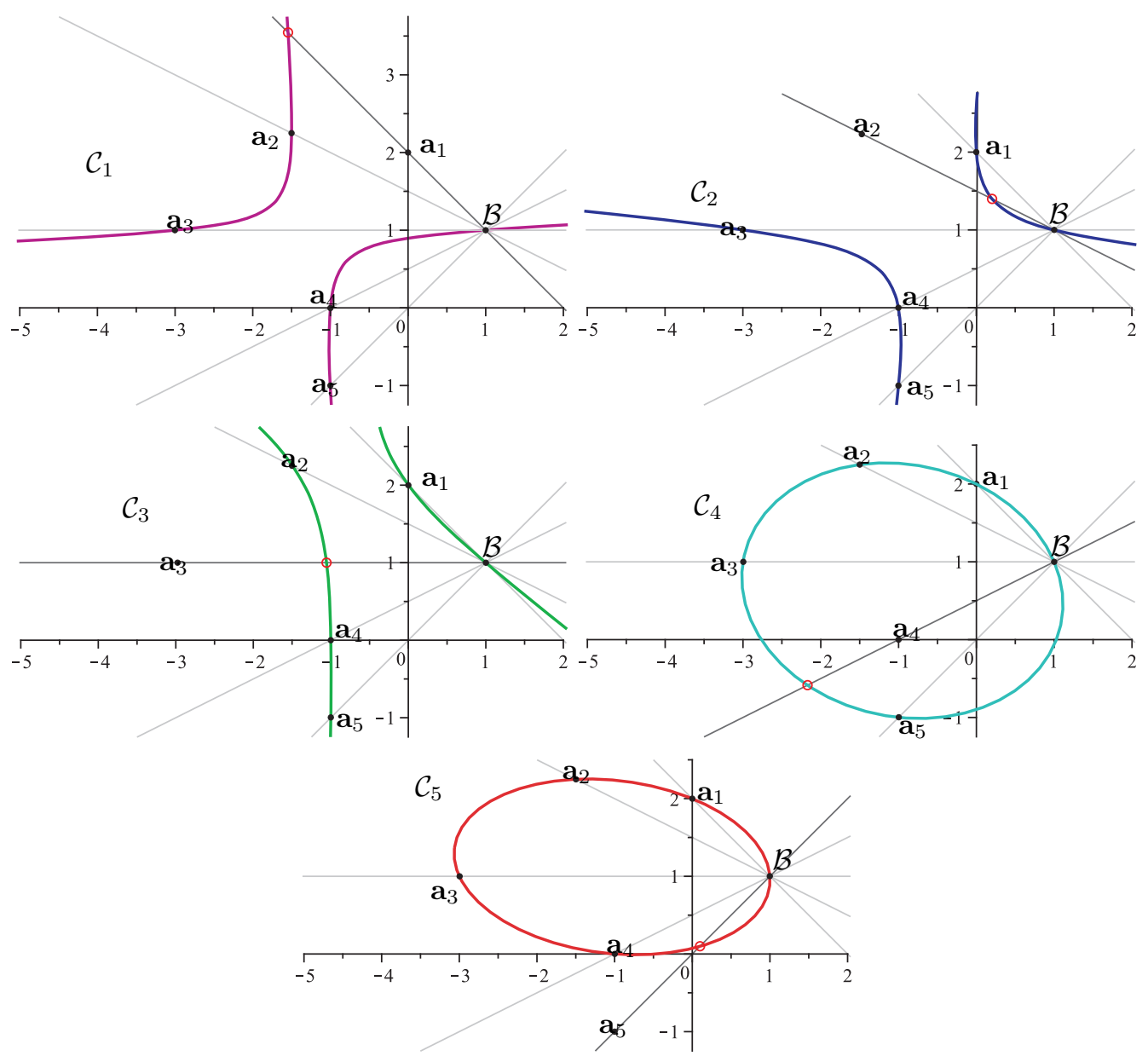

Figure 6: Singular conics, $\mathcal{C}_{i}$, for $i=1, \ldots, 5$ for the pentapod in Fig. 5.

The distance measure to an architectural singularity is given in equation (17). We use the coordinates of $\mathbf{a}_{i}$, which can be parametrized as a function of $\lambda_{i}$ as

$$
\mathbf{a}_{i}=\mathcal{B}+\lambda_{i} \frac{\mathcal{B}-\mathbf{a}_{0 i}}{\left\|\mathcal{B}-\mathbf{a}_{0 i}\right\|}, \text { for } i=1, \ldots, 5,
$$

where $\mathbf{a}_{0 i}$ is the initial location of $\mathbf{a}_{i}$, and $\mathcal{B}=(1,1,0)$, yielding

$$
\mathcal{C}\left(b_{x}, b_{y}\right)=\left(\lambda_{1} \lambda_{2} \lambda_{3} \lambda_{4} \lambda_{5}\right) \frac{K}{10}
$$

where $\lambda_{i}>0$. Any $\lambda_{i}=0$ would lead to an attachment coincident with $\mathcal{B}$, which is not an architectural singularity, but it is a non-generic case that must be studied separately. Note that the greater is the distance to the architectural singularity, the greater is the constant that multiplies the singularity polynomial, and thus, the better the pentapod will behave near a singularity. 


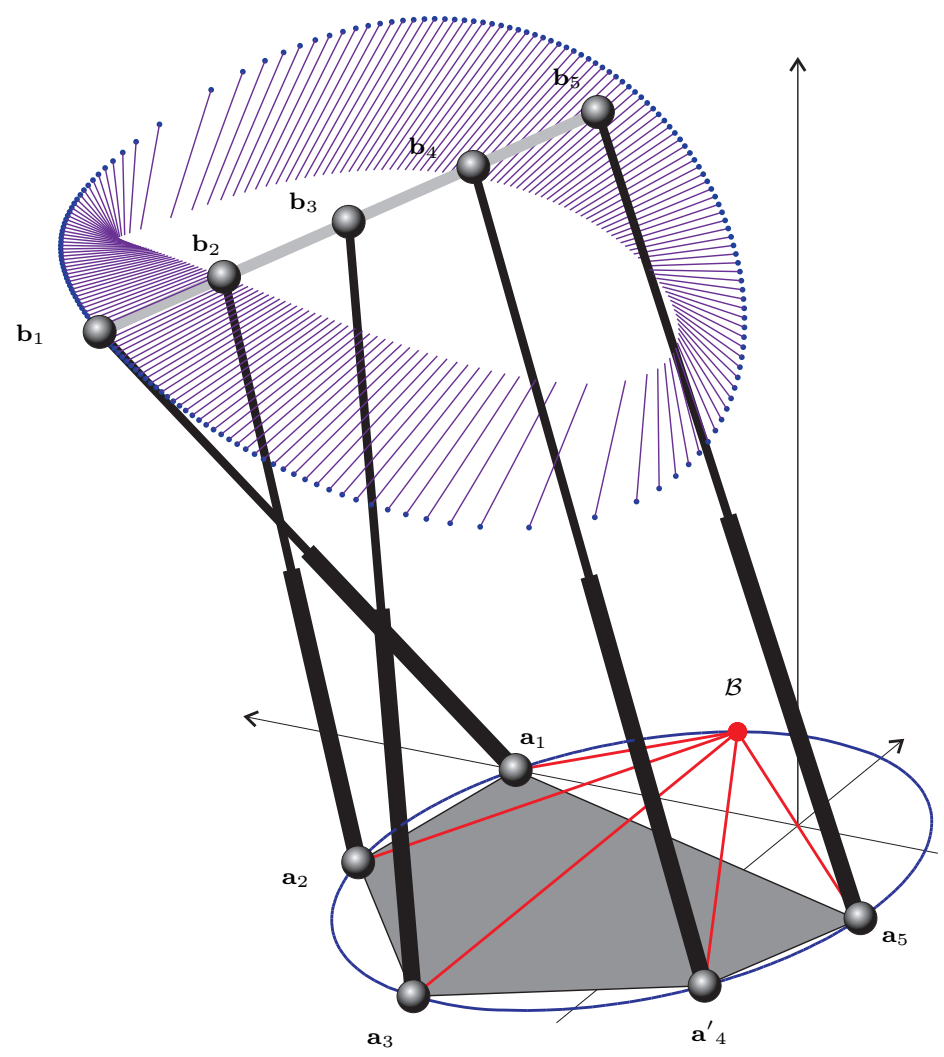

Figure 7: Self-motion of the architecturally singular pentapod analyzed in Section 4.1

$\mathcal{C}\left(b_{x}, b_{y}\right)$ is a measure of distance of point $\mathcal{B}$ to the conic defined by the 5 base attachments. Nonetheless, it can be more intuitive to measure the distance of one of the attachments $\mathbf{a}_{i}$ to its associated singular conic, that is, the conic formed by the other 4 attachments plus the point $\mathcal{B}$, i.e. $\mathcal{C}_{i}$ (defined in Section 3.2). For the current example, at the initial configuration, the five conics $\mathcal{C}_{i}, i=1, \ldots, 5$, passing through $\mathcal{B}$ and all base attachments except $\mathbf{a}_{i}$ are depicted in Fig. 6 .

Then, for instance, if attachment $\mathbf{a}_{4}$ is moved along its $\mathcal{B}$-line, the singularity locus of the analyzed robot remains unaltered, unless it is located on the conic $\mathcal{C}_{4}$ (the fourth conic in Fig. 6). In this case, the manipulator becomes architecturally singular. The new vector of coordinates for this attachment is $\mathbf{a}_{4}{ }_{4}=\left(-\frac{63}{29},-\frac{17}{29}, 0\right)$. Fig. 7 shows the self-motion of the resulting architecturally singular pentapod for a given pose with $l_{1}=4.243, l_{2}=3.786, l_{3}=4.315, l_{4}=4.893$, and $l_{5}=5.363$. The reference point $\mathbf{p}=\left(p_{x}, p_{y}, p_{z}\right)^{T}$ and the director vector $\mathbf{i}=(u, v, w)^{T}$ of $\Lambda$ are represented along the resulting self-motion. To compute this self-motion, the forward kinematics is expressed as a system of the equations dependent on two of the unknowns, one of them playing the role of a parameter, and then the equations are solved following the procedure described 


\begin{tabular}{|c|c|c|c|}
\hline $\mathrm{i}$ & $x_{i}$ & $y_{i}$ & $z_{i}$ \\
\hline \hline 1 & 2 & 0 & 0 \\
\hline 2 & $6 / 5$ & $8 / 5$ & $4 / 3$ \\
\hline 3 & $-\sqrt{2}$ & $\sqrt{2}$ & -1 \\
\hline 4 & $-\sqrt{2}$ & $-\sqrt{2}$ & 1 \\
\hline 5 & $6 / 5$ & $-8 / 5$ & $-4 / 3$ \\
\hline
\end{tabular}

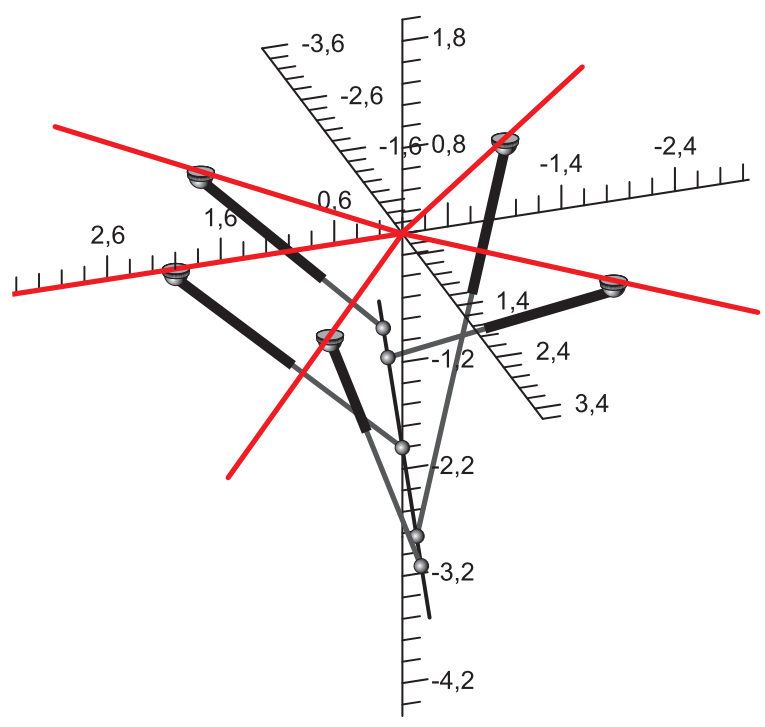

Figure 8: Coordinates of the attachments $\left[\mathbf{a}_{i}=\left(x_{i}, y_{i}, 0\right)\right.$ and $\left.\mathbf{b}_{i}=\mathbf{p}+z_{i} \mathbf{i}\right]$ and scheme of the pentapod analyzed in Section 4.2.

in $[7]^{2}$.

For the architecturally singular manipulator, the five conics $\mathcal{C}_{1}, \ldots, \mathcal{C}_{5}$ are coincident and can be computed using equation (15) and there exists a one-to-one correspondence between the moving platform attachments and the base attachments given by this unique $\mathcal{C}_{i}$-correspondence (Section 3.2).

\subsection{A radially arranged pentapod}

Thanks to the geometric interpretation of architectural singularities, it is possible to design a pentapod far from them.

In the next example $\mathcal{B}$ is located at the origin, and the $\mathcal{B}$-lines are radially arranged passing though the vertices of a pentagon.

As before, let $\lambda_{i}$ be the distance between $\mathbf{a}_{i}$ and $\mathcal{B}$. In Fig. 8 a scheme of the manipulator with coordinates appearing in the table is shown, corresponding to the configuration $\left\{\lambda_{1}=2, \ldots, \lambda_{5}=2\right\}$. For each attachment, the corresponding singular conic is depicted in Fig. 9.

For a generic configuration, the singularity polynomial is

$$
\operatorname{det}(\mathbf{J})=K\left(w\left(p_{y} w-v p_{z}\right)+p_{z}\left(p_{x} w-u p_{z}\right)\right),
$$

\footnotetext{
${ }^{2}$ The interested reader can follow the detailed computations in the Maple Worksheet attached as a multimedia material. Furthermore, included in this material, there is a video featuring the motion of the platform along its self-motion.
} 


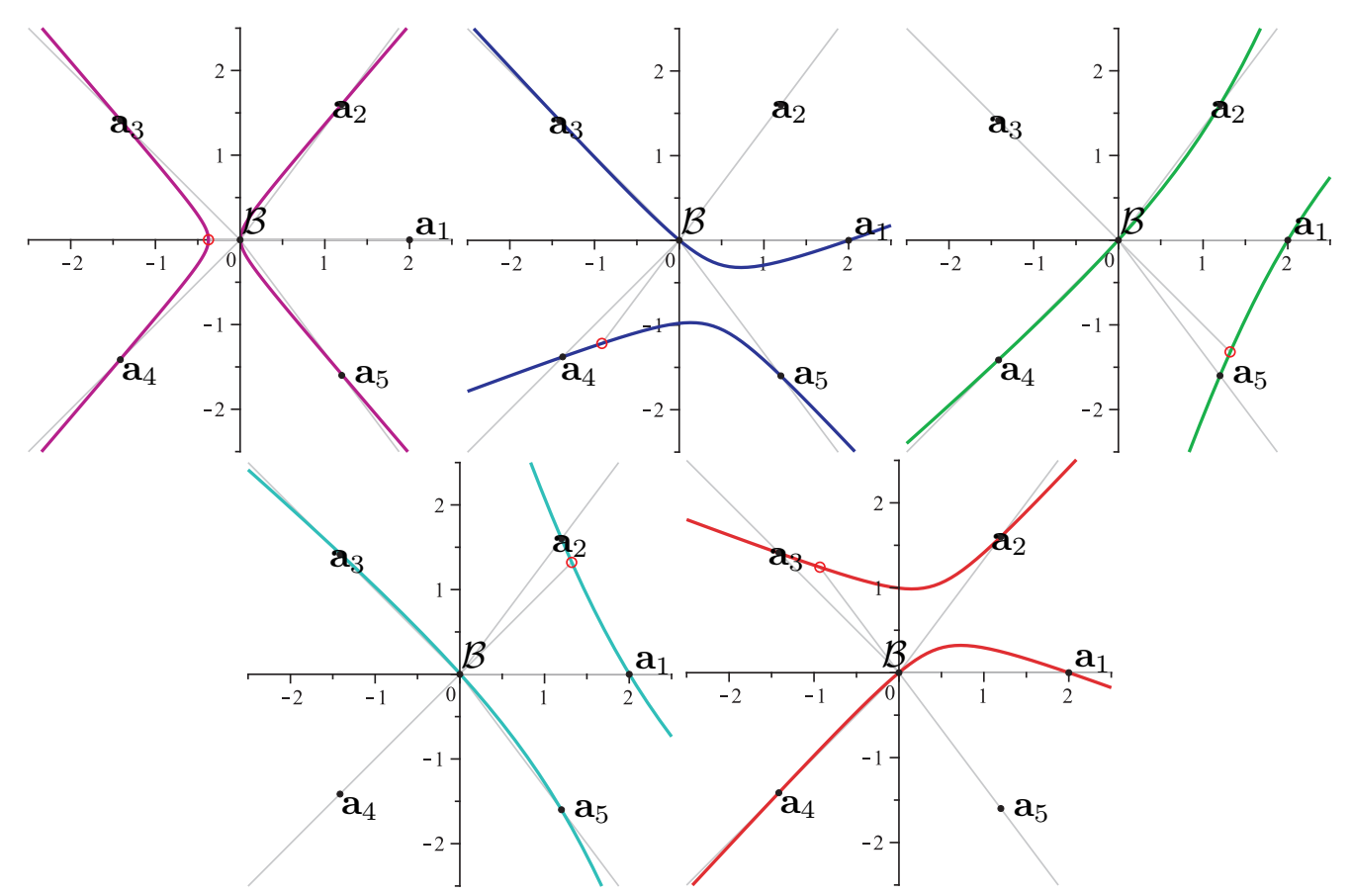

Figure 9: $\mathcal{C}_{i}$, for $i=1, \ldots, 5$, for the pentapod with attachment coordinates appearing in Fig. 8.

where

$$
\begin{gathered}
K=-\frac{2 \sqrt{2}}{225}\left(192 \sqrt{2} \lambda_{1} \lambda_{2} \lambda_{5}+42 \lambda_{2} \lambda_{3} \lambda_{5}+5 \lambda_{1} \lambda_{2} \lambda_{4}+\right. \\
5 \lambda_{1} \lambda_{3} \lambda_{5}+245 \lambda_{1} \lambda_{4} \lambda_{5}+35 \sqrt{2} \lambda_{2} \lambda_{3} \lambda_{4}+245 \lambda_{1} \lambda_{2} \lambda_{3}+ \\
\left.42 \lambda_{2} \lambda_{4} \lambda_{5}+35 \sqrt{2} \lambda_{3} \lambda_{4} \lambda_{5}+150 \sqrt{2} \lambda_{1} \lambda_{3} \lambda_{4}\right) .
\end{gathered}
$$

In other words, the cofactor values are $C_{1}=C_{2}=C_{5}=C_{6}=0, C_{3}=-K$ and $C_{4}=K$. The distance to architectural singularities defined in equation (17) is

$$
\mathcal{C}\left(b_{x}, b_{y}\right)=-\frac{9}{50} \lambda_{1} \lambda_{2} \lambda_{3} \lambda_{4} \lambda_{5} K
$$

Note that, for positive $\lambda$ 's, $K$ is always different from zero and monotone, and maximizing $\mathcal{C}\left(b_{x}, b_{y}\right)$ is equivalent to maximizing $K$. For the limits $0 \leq \lambda_{i} \leq 2$, the manipulator in Fig. 8 is the furthest one to any architectural singularity.

\subsection{A quadratically-solvable pentapod}

In [9] a family of quadratically-solvable manipulators was introduced, characterized by having point $\mathcal{B}$ at infinity and the distance between the parallel $\mathcal{B}$-lines proportional to the distances of the attachments in the platform line. The next example corresponds to one of such manipulators, with coordinates and a schematically representation given in Fig. 10. 


\begin{tabular}{|c|c|c|c|}
\hline $\mathrm{i}$ & $x_{i}$ & $y_{i}$ & $z_{i}$ \\
\hline \hline 1 & -2 & 2 & -2 \\
\hline 2 & -1 & -2 & -1 \\
\hline 3 & 0 & 3 & 0 \\
\hline 4 & 1 & -2 & 1 \\
\hline 5 & 2 & 2 & 2 \\
\hline
\end{tabular}

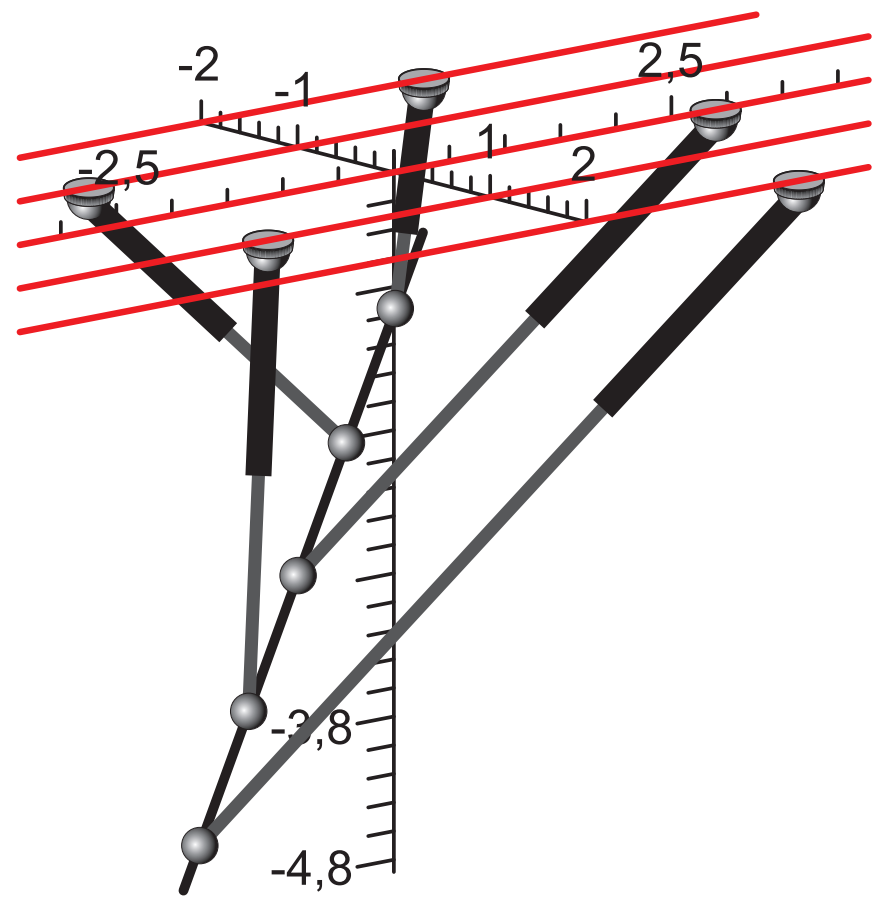

Figure 10: Coordinates of the attachments $\left[\mathbf{a}_{i}=\left(x_{i}, y_{i}, 0\right)\right.$ and $\left.\mathbf{b}_{i}=\mathbf{p}+z_{i} \mathbf{i}\right]$ and scheme of the pentapod analyzed in Section 4.3 .

The singular conic definitions established in Section 3 are also applicable when $\mathcal{B}$ is at infinity, provided that equations are handled using homogeneous coordinates. In this example, the homogeneous coordinates of point $\mathcal{B}$ are $\mathcal{B}_{H}=(0,1,0)_{H}$, which means that all conics are parabolas or hyperbolas having a vertical asymptote. In Fig. 11 the five singular conics corresponding to the attachments are plotted.

As $\mathcal{B}$ is at infinity, in this example let $\lambda_{i}$ denote the distance from each attachment to a line crossing perpendicularly all the $\mathcal{B}$-lines. By properly choosing the reference frame, all $\mathcal{B}$-lines are parallel to the $y$ axis, and the crossing line can be selected to be the $x$ axis, so that $\lambda_{i}=y_{i}$ for $i=1, \ldots, 5$.

With this parametrization, the cofactors of matrix $\mathbf{T}$ are

$$
C_{1}=-K, C_{2}=K, C_{3}=C_{4}=C_{5}=C_{6}=0,
$$

and the Jacobian matrix determinant is given by

$$
\operatorname{det}(\mathbf{T})=K\left((u-1) p_{z}-p_{x} w\right) w,
$$

where the constant factor is now

$$
\begin{aligned}
K=2\left(9 \lambda_{2} \lambda_{5}+\lambda_{5} \lambda_{4}+6 \lambda_{3} \lambda_{4}+\right. & \lambda_{2} \lambda_{1}+9 \lambda_{1} \lambda_{4} \\
& \left.-6 \lambda_{3} \lambda_{5}+6 \lambda_{3} \lambda_{2}-6 \lambda_{3} \lambda_{1}-4 \lambda_{1} \lambda_{5}-16 \lambda_{2} \lambda_{4}\right) .
\end{aligned}
$$




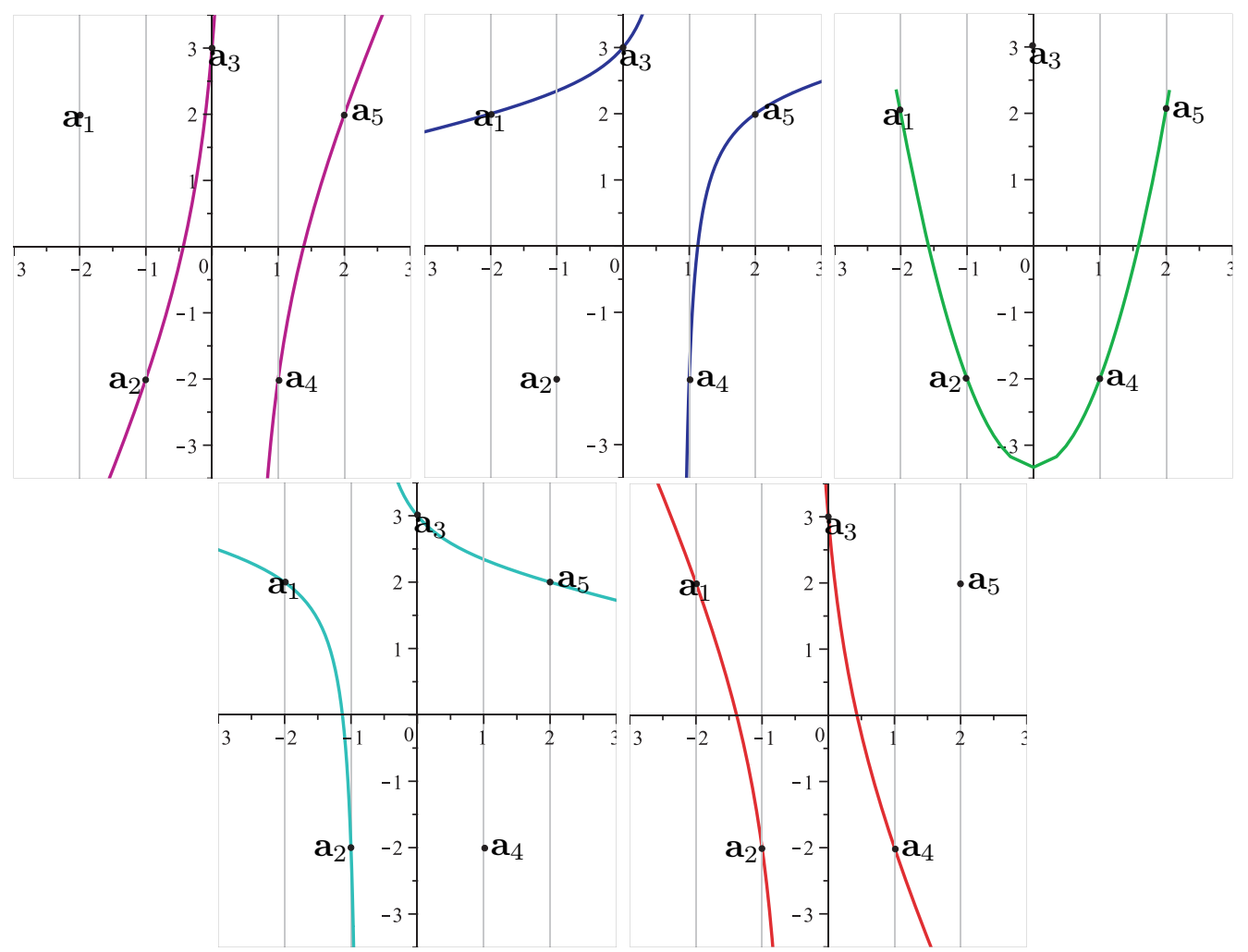

Figure 11: $\mathcal{C}_{i}$, for $i=1, \ldots, 5$, for the pentapod with attachment coordinates appearing in Fig. 10 .

In this case, the distance measure to architectural singularities given in equation (17) coincides exactly with the constant factor of the determinant:

$$
\mathcal{C}\left(B_{x}, B_{y}\right)=K
$$

Again, the configuration shown in Fig. 10 is the furthest to an architectural singularity, when $-2 \leq \lambda_{i} \leq 2$ for $i=1, \ldots, 5$.

This example is interesting because the two algebraic conditions given by Husty and Karger in [18] are satisfied, as $C_{4}=C_{5}=0$, but the manipulator is not architecturally singular. On the one hand, this is not a counter-example because it is not a generalposition case, in the sense that the coordinates of the attachments in the base and in the platform do satisfy a relation, although a non-trivial one. On the other hand, this example does show that the two algebraic conditions are only valid when leg attachments are in general position. They cannot characterize architectural singularities of non-generic pentapods, such as for example all the members of the family of quadratically-solvable defined in [9].

On the contrary, both the algebraic generalization and the geometric interpretation provided in the present paper allow to identify architectural singularities of all the pentapods with coplanar base attachments. 


\section{Conclusions}

The present work reveals that the previously defined singularity-invariant leg rearrangements are a useful tool for the complete characterization of architectural singularities, both their algebraic expression and their geometric interpretation. As a result, the pentapod manipulator, whose base and platform attachments are coplanar and collinear, respectively, is endowed with a non-trivial geometric structure consisting of a correspondence between the platform line and a pencil of lines on the base ( $\mathcal{B}$-correspondence), and five correspondences between the platform line and conics on the base $\left(\mathcal{C}_{i}\right.$-correspondences, for $\left.i=1, \ldots, 5\right)$. A distinguished point, $\mathcal{B}$, has been shown to have relevant kinematic properties.

On the one hand, the algebraic characterization of architectural singularities in terms of cofactors of the matrix $\mathbf{T}$ has been shown to be complete in comparison with previous results found in literature. On the other hand, the geometrical interpretation allows to define a measure of distance to architectural singularities, as the distance between point $\mathcal{B}$ and the conic formed by the other five attachments. This provides a useful index to be optimized in the design process, so as to obtain manipulators as far as possible from architectural singularities. This has been illustrated with several examples.

Note that the pentapod presented here can appear also as a component in a $6-\mathrm{DoF}$ Stewart-Gough platform. In this case, the singularity polynomial of the 6-legged platform factors into two terms, one representing the $5-\mathrm{DoF}$ pentapod, and the other dealing with the 6th degree of freedom. For example, a 6th leg can be added to a pentapod to gain an uncoupled rotational degree of freedom around the axial spindle platform.

The investment cost to purchase a parallel robot for a particular task could be worth if there is the possibility to reconfigure it for another task. Nevertheless, reconfiguring a parallel robot changes, in general, its kinematic equations thus converting a reconfiguration into a complex re-designing task. This would not happen using parallel manipulators whose legs can be rearranged along actuated guides following $\mathcal{B}$-lines so that the forces on its legs can be equally distributed, and its behavior for an specific task improved, while maintaining both singularities and the same forward kinematic solution. In this context, the characterization of architectural singularities carried out in this work becomes useful beyond the design process.

\section{Acknowledgments}

This work has been partially supported by the Generalitat de Catalunya through the Robotics group.

\section{References}

[1] J.-P. Merlet, Parallel Robots, Springer, 2000.

[2] B. Dasguptaa, T. Mruthyunjayab, The Stewart platform manipulator: a review, Mechanism and Machine Theory 35 (2000) 15-40.

[3] X. Kong, C. Gosselin, Classification of 6-SPS parallel manipulators according to their components, in: Proc. of ASME Design Engineering Technical Conferences, 2000, pp. DETC2000/MECH-14105.

[4] M. Wecka, D. Staimera, Parallel kinematic machine tools. current state and future potentials, CIRP Annals - Manufacturing Technology 51 (2) (2002) 671-683.

[5] G. F. Bär, G. Weiß, Kinematic analysis of a pentapod robot, Journal for Geometry and Graphics 10 (2) (2006) 173-182. 
[6] R. Neugebauer, M. Schwaar, S. Ihlenfeldt, G. Pritschow, C. Eppler, T. Garber, New approaches to machine structures to overcome the limits of classical parallel structures, CIRP Annals - Manufacturing Technology 51 (1) (2002) 293-296.

[7] C. Zhang, S. Song, Forward kinematics of a class of parallel (Stewart) platforms with closed-form solutions, Journal of Robotic Systems 9 (1) (1992) 93-112.

[8] J. Borràs, F. Thomas, C. Torras, Singularity-invariant families of 5-SPU platforms, IEEE Transactions on Robotics (Conditionally accepted).

[9] J. Borràs, F. Thomas, C. Torras, A family of quadratically-solvable 5-UPS parallel robots, in: IEEE International Conference on Robotics and Automation, 2010, pp. 4703-4708.

[10] O. Ma, J. Angeles, Architecture singularities of platform manipulators, in: IEEE International Conference on Robotics and Automation, Vol. 2, 1991, pp. 1542-1547.

[11] X. Kong, Generation of singular 6-SPS parallel manipulators, in: Proc. of ASME Design Engineering Technical Conferences, 1998, pp. DETC98/MECH-5952.

[12] K. Wohlhart, Architectural shakiness or architectural mobility of platforms, in: International Symposium on Advances in Robot Kinematics, 2000, pp. 365-374.

[13] K. Wohlhart, Mobile 6-SPS parallel mainpulators, Journal of Robotic Systems 20 (8) (2003) 509516.

[14] M. Chasles, Sur les six droites qui peuvent être les directions de six forces en équilibre, Comptes Rendus, Journal de Mathématiques Pures et Appliquées (1851) 1094.

[15] A. Dandurand, The rigidity of compound spatial grids, Structural Topology 10 (1984) 41-56.

[16] E. Borel, Mémoire sur les déplacements à trajectoires sphériques, Mémoires présentés par divers savants à l'Académie des Sciences de l'Institut National de France 33 (1) (1908) 1-128.

[17] R. Bricard, Mémoire sur les déplacements à trajectoires sphériques, Journal de l'École Polytechnique 11 (2) (1906) 1-93.

[18] M. Husty, A. Karger, Architecture singular parallel manipulators and their self-motions, in: International Symposium on Advances in Robot Kinematics, 2000, pp. 355-364.

[19] A. Karger, Architecture singular parallel manipulators, in: International Symposium on Advances in Robot Kinematics, 1998, pp. 445-454.

[20] A. Karger, Architecturally singular non-planar parallel manipulators, Mechanism and Machine Theory 43 (2008) 335-346.

[21] J. Borràs, F. Thomas, Kinematics of the line-plane subassembly in Stewart platforms, in: IEEE International Conference on Robotics and Automation, 2009, pp. 4094-4099.

[22] J. Borràs, F. Thomas, C. Torras, Singularity-invariant leg rearrangements in Stewart-Gough platforms, in: International Symposium on Advances in Robot Kinematics, 2010, pp. 421-428.

[23] J. Borràs, Singularity-invariant leg rearrangements in Stewart-Gough platforms, Ph.D. thesis, CSICUPC, to appear (2011). 